International Journal of Latest Trends in Engineering and Technology

Vol.(8)Issue(1), pp.558-565

DOI: http://dx.doi.org/10.21172/1.81.073

e-ISSN:2278-621X

\title{
VARIOUS BROADCAST DISCOVERY METHODS FOR MOBILE ADHOC NETWORK: A SURVEY
}

\author{
Dr.B.Sundaravadivazhagan ${ }^{1}$ and R.Sankari ${ }^{2}$
}

\begin{abstract}
Mobile Ad-Hoc Networks (MANET) is comprised of mobile nodes that are self- organizing infrastructure less network establishing connected via multihop wireless links. Broadcasting is the important concept in the communication technology, which utilize the mobile ad hoc network while forwarding the message from one node to another node. During the information transmission, the high broadcast traffic increases the contention, collision problem. So, this paper reviews the different broadcast discovery approaches like Route Discovery, Link State Based Route Discovery for Broadcast Approach, are for reducing the broadcast problems present in the network. Further, the paper shows that different protocols for broadcasts also discussed.
\end{abstract}

Keywords - Broadcasting, Link State Based Route Discovery for Probabilistic Broadcast Approach, MANET.

\section{INTRODUCTION}

Mobile Ad Hoc Network (MANET) is a base free system with wireless mobile nodes. MANET is a sort of Ad Hoc coordinates with special qualities like open system limit, dynamic topology, distributed system, quick and brisk execution then hops-by-hop interchanges [1]. Mobile networking is a standout amongst the most critical advances supporting inevitable process. Amid the last decade, the progresses in both hardware and programming systems have brought about portable hosts and remote systems administration regular and incidental [2]. By and large there are two particular methodologies for authorizing wireless mobile units to speak with each other: Infrastructure is a wireless mobile system have generally been founded on the cell idea and depended on the great base support, in which mobile phones speak to get to focuses like base stations associated with the established system base. Average cases of these sorts of remote systems are GSM, UMTS, WLL, WLAN, and so on.

Predictable wired systems have been the fundamental center of research. Though, they are valuable, yet not appropriate for versatile circumstances. At the point when cell phones, for example, scratch pad and PDAs showed up, clients needed remote availability, and thus properly turn into a reality. Remote systems might be framework situated, as in traditional WiFi [2] or foundation less as in Mobile Ad-hoc Networks (MANETs) [3]. One of the predominant starting inspirations for MANET innovation originated from military applications in situations where there is no foundation. Be that as it may, while such applications stay vital, MANET investigate has differentiated into territories, for example, fiasco alleviation, sensor systems, and individual area methods [3].

MANET contains a self-sufficient cluster of portable users that speak with moderate wireless connections. Because of the versatility of hubs, expedient and unusual changes might be made over the time. In such systems, the versatile hubs keep up all the system exercises like course disclosure and message transmission.

Broadcasting is the synchronous transmission of comparable messages to different recipients. In systems administration, broadcasting happens when a transmitted data packet is gotten by all system devices.. Security issues

1 Department of Computer Science and Engineering Renganayagi Varatharaj College of Engineering, Thayalpatti(via), salavarpatti, India

2 Department of Computer Science and Engineering Renganayagi Varatharaj College of Engineering, Thayalpatti(via),salavarpatti, India 
may develop in the midst of broadcasting and prompt information misfortune if a system is assaulted by intruders. Uses of MANETs happen in conditions like war regions or real hazardous situations where systems should be sent promptly, however base stations or altered system buses are not accessible. Unicast directing in MANET has been concentrated on in a few articles [1,3,5]. A working gathering called "Manet" has been shaped by the Internet Engineering Task Force (IETF) to examine the related issues and authorize the request about in MANET [3].

Broadcasting is a typical operation in frequent applications, e.g., chart related issues and dispersed figuring issues. It is likewise generally used to determine many system layer issues. In a MANET specifically, because of host portability, broadcastings are relied upon to be performed all the more as often as possible (e.g., for paging a specific host, sending an alert flag, and finding a course to a specific host. Broadcasting may likewise be utilized as a part of LAN copying [6] or serve if all else fails to give multicast benefits in systems with quick evolving topologies.

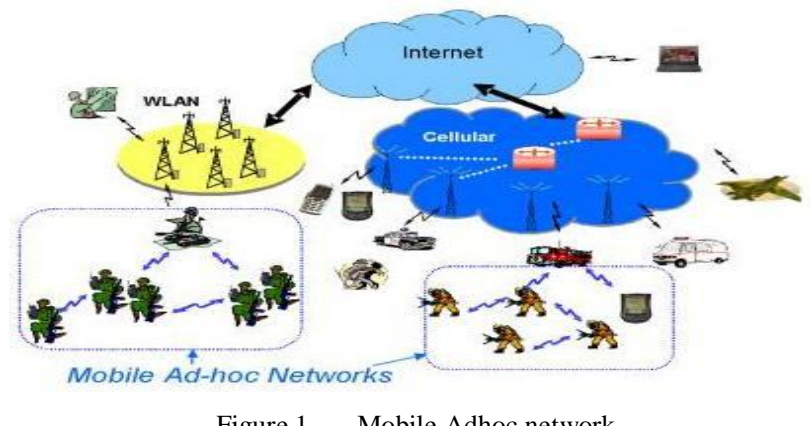

Figure 1. Mobile Adhoc network

\section{LITERATURE REVIEW}

S. Priyadsrini, et, al., (2012) proposed a Route Discovery in Manets with Improved Route Lifetime. Actualize our proposed algorithms in AODV and the execution is assessed against the first AODV. Our convention enhances the system execution and diminishes the calculation overhead by evading incessant course revelation since select a steady way with long lifetime [7]. With the assistance of network simulator and substantiate that proposed convention performs superior to the current strength based steering conventions with enhanced packet conveyance proportion and decreased routing overhead.

Hung-Chin Jang, Chih-Chia, (2010) proposes a Directive Location-Aided Routing (DLAR) protocol to select the neighbor nodes based on the mobility direction of the source node to prevent the broadcast storm[8]. Furthermore the route discovery process is achieved by employing the AODV for Location Aided routing and uses the local repair procedure to initiate path discovery at the local disconnected location in order to fast up the path discovery process. The performance of the protocol is measured in terms of RREQ packet capacity, packet delivery ratio and so on and it is compared with the existing protocols like AODV, DSR and LAR. The simulation results show that the DLAR protocol achieves a better performance than the existing protocols.

Kalani, G, et, al., (2014) proposed a 2-hop neighbor based protocol in order to reduce a broadcast storm in MANET by selecting the minimum number of hops using self-pruning and dominant-pruning. The Network coding idea (COPE) is used in order to overcome the number of transmissions by victimization using a logical operation [9]. A new effort has been made in the process by introducing an ant colony optimization to the COPE protocol with pruning algorithm. The pheromone value is used to decide the packet combination and this value is estimated based on the intersection of the forward node packet list by pruning algorithm and the sender packet list. The experimental results show that the protocol has the best capability to reduce the broadcast storm.

Khalaf, M.B, et, al., (2010), proposed a dynamic probabilistic broadcasting algorithm for the Broadcast storm problem in MANET[10]. The approach improves by including the well-known Ad hoc On demand Distance Vector Protocol (AODV). The simulation results show that the proposed approach outperforms better than the existing approaches.

Xin Ming Zhang, et, al., (2013) proposed a neighbor coverage based probabilistic approach for broadcast problem. A rebroadcast delay has been proposed to find the rebroadcast order and then the additional coverage ratio has been accurately obtained by sensing coverage knowledge. A connectivity factor has been defined to offer the node density adaptation. A rebroadcast probability has been set by combining the connectivity factor and additional coverage ratio[11]. Thus the approach takes the advantage of the probabilistic technique and neighbor coverage ratio and the results shows that the approach can reduce the retransmission significantly by enhancing the routing performance.

Xi Zhou, et, al., (2011) proposed lightweight, reliable service discovery approach uses the basis as a Service Magnetic Field (SMF) concept and a cross layer is implemented to integrate the SMF and AODV routing 
protocol[12]. The SMF determines the more efficient service providers to the consuming node and in additionally it provides information about the direction for the service discovery and it ultimately reduces the number of broadcast packets. The experimental results show that the approach is effective than the previous approaches.

Aminu Mohammed, et,al., (2007) proposed a broadcasting scheme by integrating the advantages of traditional probabilistic and counter-based schemes to minimize the broadcast storm in the MANET network[13]. The simulation result shows that the system achieves a significant performance in terms of reachability, rebroadcast and latency.

Muneer Bani Yassein, et, al., (2013) proposes an adaptive algorithm to reduce the overall network overhead with minimizing the broadcast storm problem. In additionally, the performance of the different traffic types such as Traffic Gen and Constant bit rate (CBR) has been evaluated. The simulation results show that the algorithm enhanced the overall network performance[14]. The evaluation of the traffic type concludes that the Traffic GEN is outperforming in terms of throughput and delay values than CBR traffic.

Abdelfettah Belghith, et, al., (2012) proposes a proactive routing framework based on a generic model and probabilistic decisions to evaluate the existence probabilities of links and nodes. In additionally, a distributed algorithm has been presented to gather the cartography of the network. This cartography is utilized to instantiate the presence probabilities[15]. The simulation results show that the scheme outperforms better than the conventional routing protocols.

Kaur, G, Garg, R, (2014), proposed a scheme to reduce the broadcast storm problem and the scheme takes the advantage of techniques such as neighbor knowledge, particle swarm optimization and probabilistic rebroadcast. The order of forwarding is decided by calculating the rebroadcast delay. The rebroadcast probability is determined by the neighborhoods covered seat. The probability of rebroadcast is determined by comparing the rebroadcast probability with the value of the particle swarm optimization function[16]. The simulation results show that the scheme has the capability to reduce the broadcast storm than the existing approaches.

Khalaf M.B, Al-Dubai, (2012) proposed velocity aware route discovery approach to mitigate the broadcast storm problem. The approach excludes the unstable nodes while during the route discovery process. The simulation results show that the approach performs better than the existing approaches in terms of link stability and RREQ packet overhead. (Shuhui Yang, Jie Wu, 2010) proposed a scheme using a directional antenna for efficient broadcasting in MANET. The network coding is used to reduce the number of transmissions. The forwarding nodes are selected using the directional antenna for transmitting the coded messages to the predefined sectors[17]. The simulation results show that the scheme reduces the number of transmissions in the broadcast application.

Kalani, G, et, al., (2014) proposed a 2-hop neighbor based protocol in order to reduce a broadcast storm in MANET by selecting the minimum number of hops using self-pruning and dominant-pruning. The Network coding idea (COPE) is used in order to overcome the number of transmissions by victimization using a logical operation [18]. A new effort has been made in the process by introducing an ant colony optimization to the COPE protocol with pruning algorithm. The pheromone value is used to decide the packet combination and this value is estimated based on the intersection of the forward node packet list by pruning algorithm and the sender packet list. The experimental results show that the protocol has the best capability to reduce the broadcast storm.

\section{MANET ROUTING DISCOVERY PROTOCOLS}

\subsection{Route discovery:}

There are two primary ways to deal with steering in MANETs: Receptive routing and proactive navigation. Responsive Routing lessens overhead to the detriment of postponement in finding a reasonable cause; though, the turnaround is valid for proactive directing. For QoS-mindful routing, another issue is deciding the mix of lessened idleness and decreased overhead that is best to support QoS. At the point when a hub needs to speak with another hub in the system an interesting correspondence way is built up between the sender and the recipient hubs. The source hub checks the area vector for the goal. In the event that the goal hub is distinguished to be the single jump neighbor of the source, the source hubs begins transmitting information bundles. The transmission of information will be continued until there is no adjustment in the geological positions of the source and the goal hubs.

The executed in the Ad Hoc On-Demand Distance Vector (AODV) convention as it is one of the prominent of request directing conventions, and it is the joint convention of Distance Source Distance Vector (DSDV) and Dynamic Source Routing (DSR). Be that as it may, this directing convention was not considering the course lifetime and Quality of service (QoS) parameters amid course disclosure. In our altered AODV convention, the source which needs to find the course to a specific goal advances Route REQuest (RREQ) parcel. The middle hubs figure hub lifetime and association lifetime utilizing the calculations as portrayed as a part of the above area and it advances the course, ask for alongside the processed data to the next hub till it achieves the goal. After RREQ achieves the goal, it 
advances Route REPly (RREP) to the source. Each middle of the road hub trade two control parcels. So every hub computes the association lifetime after it got the relating RREP. The Route Lifetime is figured as the base of the hub and connection lifetime and it is sent alongside RREP to the source through the middle of the road hubs. The source chooses the way with the longest course lifetime.

The neighbors in the area vector are put away in the expanding request of their separations. The source hub creates a RREQ bundle and advances it to $\mathrm{n} / \mathrm{k}$ neighbors (where $\mathrm{n}$ is the aggregate number of the RREQ 4 neighbors and K1 reachability parameter - an irregular number somewhere around after the range vector focusing on the most distant hubs from the source hub. The planned neighbors check their neighborhood vectors and find the goal else similar system is rehashed till the goal is found.

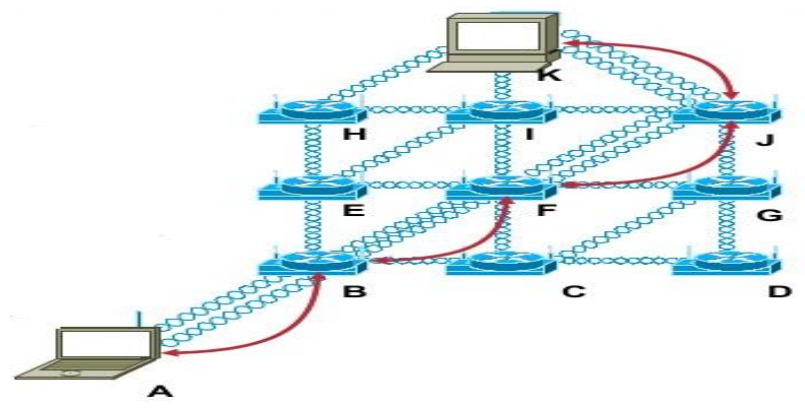

Figure 2. Detected source route (A, B, F, G, K)

\subsection{Link state based route discovery approach}

The result of the transfer storms is the overwhelming activity conflict and in this manner impacts of parcels in light of the immense flooding of transfers between neighboring hubs where colossal control overhead will be the outcome. In this study, a link state based course disclosure approach is proposed which is a probabilistic approach and expect to diminish the communicate fasten keeping in mind the end goal to lessen the communicate is decreased after the underlying transmission with a pre-decided link state so that each hub keeps up the one jump interface state data to rebroadcast the message of RREQ and selecting viable link will diminish the control overhead which eventually diminish the transfer strom in the proposed approach.

\subsubsection{Link State Routing Protocol}

A link state routing protocol is one among the two fundamental classes of routing protocol utilized as a part of packet switching and other is a separation vector routing protocol.

Examples of link-state routing protocols conventions grasp open most brief way first (OSPF) and a halfway system to middle of the road system (IS-IS). The link state protocol is performed by every exchanging hub in the system, its utilization of prepared sending parcels inside the switches. The crucial origination of link-state routing protocols is that every hub develops a guide of the property to the system, inside the type of a diagram, demonstrating that hubs are associated with those individual hubs. Each hub, then freely figures back to back best legitimate way from it to every conceivable goal inside the system. The social event of best way can then sort the hub's directing table. Link state algorithms are in some cases shown informally as each switch telling the world in respects to its neighbors.

Optimized Link State Routing-based Routing. The incorporated QoS highlights into the Optimized Link State Routing (OLSR) protocol to discover a course with bigger data transmission (OLSR-based). This approach does not alter the directing plan of OLSR, but rather it picks diverse criteria that join transmission capacity into thought to choose the multipoint hand-off (MPR) set in order to locate a bigger transfer speed course. Transfer speed estimation is performed by exploiting the bearer sensory ability in the IEEE 802.11 MAC convention and measuring the rate of occupied time to get the accessible data transmission data. Path support and resource reservation are not considered in this protocol.The Optimized Link State Routing (OLSR) is a table-driven, proactive routing protocol produced for MANETs. It is an optimization of unadulterated link state protocols that decreases the extent of control packages and the quantity of control packet transmissions required. OLSR lessens the control activity overhead by utilizing Multipoint Relays (MPR), which is the key thought behind OLSR. An MPR is a hub one-jump neighbor, which has been sent packets. Rather than perfect flooding of the system, packages are sent by hubs MPRs. This delimits the system overhead, hence being more effective than immaculate link state routing protocol. OLSR is 
appropriate to substantial and thick mobile systems. As a result of the utilization of MPRs, bigger and more thick a system, the more upgraded link state routing is accomplished. MPRs assist giving the most limited way to a goal. The main necessity is that all MPRs proclaim the link data for their MPR selectors (i.e. The hubs which have picked them as MPRs). The system topology data is kept up by occasionally trade interface state data. On the off chance that more reactivity to topological changes is required, the time interim for trading of connection state data can be diminished. Control messages OLSR utilizes three sorts of control messages: HELLO, Topology Information (TC), and Multiple Interface Declaration (MID). A Hello message is sent intermittently to the greater part of the hub's neighbors. Hi messages contain data around a hub's neighbor, the hubs it has picked as MPRs (i.e., the MPR Selector set), and a rundown of neighbors with whom bidirectional links have not yet been affirmed.

\subsubsection{Optimized Link State Routing (OLSR) Protocol}

This protocol is based upon a link state algorithm and is a indicate point routing protocol. In this protocol, every hub keeps up the data with respect to the topology and is traded occasionally. The fundamental preferred standpoint of OLSR is that it decreases the span of control message and accordingly minimizes the quantity of rebroadcasting hubs by utilizing multipoint answering methodology. At the point when the topology changes, every hub chooses its neighboring hubs to retransmit the information. These arrangements of hubs are called as multipoint transfers for that hub. OLSR utilizes two sorts of control messages, i.e. "hi" message and "topology control" message. Hi messages are for the status of connection and host's neighbors. While the topology control messages are for its own neighbors and utilized for broadcasting data.

\subsubsection{Link State Routing Protocols}

Link State protocols, otherwise called briefest way first or circulated database protocols, are working around a notable algorithm of graph theory, E.W. Dijkstra's most brief way first algorithm. Interface State conventions act like a guide. Every switch shares its connection data as a Link State Advertisement (LSA), or Link State PDU (LSP). A link state switch utilizes interface state data to make a topology delineate to choose the best way to the goal in the topology. LSAs proliferates to each neighbor switch utilizing the protocol particular multicast address, every switch that gets the LSA, overhauls its Link-State-Database(LSDB) and advances the LSA to its neighbor switches inside an area. SPF tree is then connected to the LSDB to locate the best way to achieve the goal and the best way is then added to the steering table. A case representation demonstrating join state path protocols, and separation vector convention is demonstrated as follows:

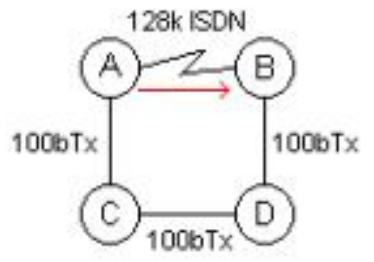

A. Distance vector

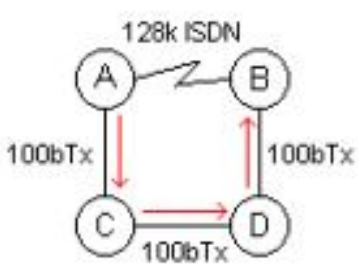

B. Link state

3 Figure. $\quad$ Link State Routing Protocols

\section{BROADCASTING IN A MANET}

A MANET comprises of an arrangement of mobile hosts that may speak with each other every now and then. No base stations are bolstered. Every host is outfitted with a CSMAICA (bearer sense different access with impact evasion) handset. In such environment, a host may speak with another, straightforwardly or by implication. In the last case, a multihop situation happens, where the bundles began from the source host are transferred by a few middle of the road has before achieving the goal. The broadcast problem alludes to the sending of a message to different has in the system. The issue considered here has the accompanying qualities.

The broadcast is spontaneous.

Any mobile host can issue a broadcast operation whenever. For reasons, for example, the host portability and the absence of synchronization, setting up any sort of worldwide topology information is restrictive (truth be told, this is in any event as hard as the communicate issue). Practically no nearby data might be collected ahead of time. 
The broadcast is untrustworthy.' No confirmation, system will be utilized. However, an endeavor ought to be made to convey a broadcast message to whatever number has as could reasonably be expected without paying an excessive amount of exertion. The inspirations to make such a presumption are (i) a host may miss a broadcast message since it is disconnected, it is incidentally disengaged from the system, or it encounters redundant impacts, (ii) affirmations may bring about genuine medium dispute (and along these lines another "tempest") encompassing the sender, and (iii) in numerous applications (e.g., the course disclosure in a $100 \%$ dependable broadcast is pointless.Also, accept that a host can distinguish copy broadcast messages. This is key to forestall unending flooding of a message. One approach to do as such is to take up with every communicate message a tuple (source ID, arrangement number) as that in . At long last, we remark that we don't bind ourselves to the telecom of similar message. 3

In this paper, concentrate on the flooding conduct in MANET - the wonder where the transmission of a parcel will trigger other encompassing hosts to transmit the same (or changed) bundle. Should demonstrate that if flooding is utilized aimlessly, numerous repetitive messages will be sent and genuine conflict/crash will be acquired. Will probably tackle broadcast on account of effectiveness.

\section{Broadcast Storm, Caused by Flooding}

A direct way to deal with perform, broadcast is befitting. A host, on getting a broadcast message surprisingly, has the commitment to rebroadcast the message. Distinctly, this costs $n$ transmissions in a system of it hosts. In a CSMAICA organize, downsides of flooding include:

Redundant rebroadcasts:

At the point when a portable host chooses to rebroadcast a broadcast message to its neighbors, every one of its neighbors as of now have the message.

Contention

After a mobile host broadcast a message, if a large number of its neighbors choose to rebroadcast the message, these transmissions (which are all from adjacent hosts) may extremely battle with each other.

Collision

Since of the inadequacy of backup mechanism, the absence of RTSKTS discourse, and the nonappearance of CD, impacts will probably happen and cause more destruction.

\section{Simple Flooding}

The most straightforward strategy for broadcasting is flooding. In this system, every hub retransmit the RREQ when gotten interesting. Parcels that have as of now been gotten are just dropped. Flooding presents an expansive number of repetitive messages and prompts dispute and the impact which is alluded to " broadcast storm issue".

Probabilistic Scheme

In likelihood based techniques, every hub is appointed a likelihood for retransmission. At the point when a hub gets a broadcast message interestingly, the hub rebroadcasts the message with a likelihood P. For scanty systems, the estimation of $\mathrm{P}$ must be sufficiently high to encourage a higher packet delivery ratio. At the point when $\mathrm{P}=1$, the plan resorts to basic flooding.

\section{Counter-based Scheme}

A broadcast message got interestingly, is not promptly retransmitted to the area. The message is lined up for a period called the Random Assessment Delay (RAD) amid which the hub may get a similar message (repetitive broadcasts) from some of its different neighbors. After the RAD clock lapses, if the quantity of times similar message is gotten surpasses a counter edge, the message is not retransmitted and is essentially dropped.

Area-based Methods

In the region based techniques, a typical transmission range is expected and a hub will rebroadcast if just adequate new region can be secured with the retransmission.

Distance-based Scheme

At the point when a hub gets a formerly inconspicuous communicate messages, the hub figures the separation amongst itself and the sender. On the off chance that the sender is nearer than a limit separation, the message is dropped. Something else, the got the message is reserved and the hub starts a Rapid application development (RAD) clock. Repetitive communicate messages got before the expiry of the RAD clock are likewise reserved. At the point when the RAD clock lapses, the hub figures the separation amongst itself and the neighbor hubs that already communicate the specific message. In the event that any such neighbor hub is nearer than an edge remove esteem, the message is dropped. Something else, the message is retransmitted. Area based Scheme Whenever a hub begins or rebroadcasts a message, the hub puts its area data in the message header. The receiver hub, data the extra scope range that would be reachable if it somehow managed to rebroadcast. In the event that the extra scope is not exactly a limit esteem, every future gathering of similar message will be dropped. Something else, the RAD clock is begun. 
Repetitive broadcast messages got before the expiry of the RAD clock are additionally reserved. After the RAD clock terminates, the hub considers all the stored messages and recalculates the extra possible scope region if it somehow happened to rebroadcast the specific message. In the event that the extra possible scope range is not exactly an edge esteem, the stored messages are dropped. Generally the message is rebroadcast.

\begin{tabular}{|c|c|c|c|}
\hline Metrics & Route discovery & Link state based route discovery & Broadcast discovery \\
\hline RoutingOverhead & Less & Less & Less Very less \\
\hline Delay & Average & Less & Less \\
\hline Packet Loss & High & Less & Very less \\
\hline Cost & No cost consideration & ------ & Less \\
\hline
\end{tabular}

\section{V.CONCLUSION}

This paper reviews the different broadcast discovery approaches like Route Discovery, Link State Based Route Discovery for Broadcast Approach, are for reducing the broadcast problems present in the network. Further, the paper shows that different protocols for broadcasts also discussed. Varies routing schemes are optimized in literatures. Routing protocols are used to have the knowledge about the neighbor covered nodes and to generate the less traffic in the routing. Hence there exists a need to find an efficient path while during the route discovery phase with the lowest number of broadcasts to reduce the broadcast storm problem.

\section{REFERENCE}

[1] Ali Dorri and Seyed Reza Kamel and Esmail kheyrkhah," Security Challenges In Mobile Ad HocNetworks: A Survey", International Journal of Computer Science \& Engineering Survey (IJCSES) Vol.6, No.1, February 2015.

[2] Jun-Zhao Sun," Mobile Ad Hoc Networking: An Essential Technology for Pervasive Computing", International Conferences on Info-tech \& Info-net, Beijing, China, C:316 - 321. Sun J (2001).

[3] Saleh Ali K. Al-Omari 1, Putra Sumari," An overview of mobile ad hoc networks for the existing protocols and application", international journal on applications of graph theory in wireless ah hoc networks and sensor networks (Graph-Hoc), Vol.2,No.1, March 2010.

[4] Zhenqiang Ye, Srikanth V. Krishnamurthy, Satish K. Tripathi,” A Framework for Reliable Routing in Mobile Ad Hoc Networks", IEEE INFOCOM 2003.

[5] Ashish Kumar, M. Q. Rafiq, M. Q. Rafiq," Performance Evaluation of Energy Consumption in MANET", International Journal of Computer Applications (0975 - 8887) Volume 42- No.2, March 2012.

[6] Ritu Parasher1, Yogesh Rathi2," A_AODV: A Modern Routing Algorithm for Mobile Ad-Hoc Network", International Research Journal of Engineering and Technology (IRJET), Volume: 02 Issue: 01 | Apr -2015.

[7] S. Priyadsrini, T. M. Navamani, and Venkatesh Mahadevan," An Efficient Route Discovery in Manets with Improved Route Lifetime", International Journal of Information and Electronics Engineering, Vol. 2, No. 4, July 2012.

[8] Hung-Chin Jang, Chih-Chia Hung, "Direction based routing strategy to reduce broadcast storm in MANET", International conference on Computer, IEEE, pages 445-450, 2010.

[9] Kalani, G, Srinivas K, Nagaraju A, "Adaptive ant colony network coding to neighbor topology based broadcasting techniques in MANETs", International Conference on Advances in Computing, Communications and Informatics, IEEE, pages 2163-2167, 2014.

[10] Khalaf M.B, Al-Dubai A.Y, Buchanan W, "A new adaptive broadcasting approach for mobile ad hoc networks", International conference on Wireless Advanced, IEEE, pages 1-6, 2010.

[11] Xi Zhou, Yifan Ge, Xuxu Chen, Yinan Jing, Weiwei Sun, "SMF: A Novel Lightweight Reliable Service

[12] Discovery Approach in MANET", International Conference on Wireless Communications, Networking and Mobile Computing, IEEE, pages 1-5, 2011.

[13] Aminu Mohammed, Mohamed Ould-Khaoua and Lewis Mackenzie "An Efficient Counter-Based

Broadcast Scheme for Mobile Ad Hoc Networks", Formal Methods and Stochastic Models for Performance Evaluation, Springer, vol 4748, pages 275-283, 2007.

[14] Muneer Bani Yassein, Balqees Abd Hameed, Wail Mardini, Yaser Khamayseh,“ Performance Analysis of Adjusted Counter Based Broadcasting in Mobile Ad Hoc Networks", Communication and Network, Scientific Research, vol 5, pages 353-359, 2013.

[15] Abdelfettah Belghith, Mohamed Amine Abid, and Adel BenMnaouer, "Adaptive Probabilistic Proactive 
Routing for Dense MANETs", Journal of Computer Networks and Communications, Research article, article id 234824, vol 2012, 2012.

[16] Kaur, G, Garg, R, "NCPR-PSO neighborhood coverage probabilistic rebroadcast with particle swarm optimization", International Conference on Confluence, The Next Generation Information Technology Summit (Confluence), IEEE, pages 298-303, 2014.

[17] Khalaf M.B, Al-Dubai A.Y, Abed M, "New velocity aware probabilistic route discovery schemes for

Mobile Ad hoc Networks", International Conference on Software Telecommunications and Computer Networks, IEEE, pages 1-6, 2012.

[18] Xin Ming Zhang, En Bo Wang, Jing Jing Xia, and Dan Keun Sung, “ A Neighbor Coverage-Based

Probabilistic Rebroadcast for Reducing Routing Overhead in Mobile Ad Hoc Networks", International Conference on Mobile Computing, IEEE, vol 12, no 3, 2013. 